\title{
ZonaTIC: Sitio web para la divulgación de la ciencia de las TIC en pro de la inclusión digital
}

\section{ZonaTIC: Website for the outreach of ICT science for digital inclusion}

\author{
TREJO-CUEVAS, Apolinar†*, PARRA-RODRÍGUEZ, Gerardo, DÍAZ-ROBLEDO, María Teresa y \\ TORRES-YÁÑEZ, Javier Jesús
} Universidad Tecnológica del Norte de Guanajuato. Av. Educación Tecnológica No. 34, Fracc. Universidad, Dolores Hidalgo
C.I.N., Guanajuato, México

ID $1^{\mathrm{er}}$ Autor: Apolinar, Trejo-Cuevas / ORC ID: 0000-0001-9977-9226

ID $1^{\text {er }}$ Coautor: Gerardo, Parra-Rodríguez / ORC ID: 0000-0002-6389-3227

ID $2^{\text {do }}$ Coautor: María Teresa, Díaz-Robledo / ORC ID: 0000-0001-5816-5113

ID $3^{\text {er }}$ Coautor: Javier Jesús, Torres-Yáñez / ORC ID: 0000-0003-1313-9477

DOI: $10.35429 / J I T .2019 .20 .6 .14 .18$

Recibido: 10 de Junio, 2019; Aceptado 30 de Septiembre, 2019

\section{Resumen}

Objetivo. Implementar un sitio web que permita divulgar la ciencia de Tecnologías de la Información y Comunicación al personal de la comunidad universitaria de la UTNG para facilitar la inclusión digital, aprovechando la infraestructura actual con la que cuenta la universidad, así como de su capital humano. Metodología. El modelo de desarrollo adoptado fue un modelo concurrente: Para el desarrollo Frontend se empleó el modelo espiral, el cual facilita su diseño por medio las etapas de elaboración de prototipos, análisis y diseño. Por otra parte, para el desarrollo del Backend se hizo uso del modelo de cascada, dada la naturaleza proyecto, en donde los requerimientos son preestablecidos y con pocos cambios. Las fases de este modelo son: (Requisitos. Levantamiento de requerimientos, Diseño. Elaboración de la base de datos y diagramas de clases, Implementación. Codificación, Verificación. Pruebas, Mantenimiento. Corregir errores en la funcionalidad) Contribución. Implementación del aprendizaje basado en proyectos (ABP) como una estrategia didáctica motivadora en el desarrollo de aplicaciones web. Utilización de herramientas de desarrollo robustas empleadas hoy en día por las fábricas de software, tales como: Java, Spring, HTML5, CSS3, y Apache Maven; todo ello bajo una arquitectura Modelo Vista Controlador.

Aprendizaje basado en proyectos, TIC, Desarrollo web

\begin{abstract}
Objective. Implement a website that allows the outreach of the science of Information and Communication Technologies to the staff of the university community of the UTNG to facilitate digital inclusion, taking advantage of the current infrastructure that the university has, as well as its human capital. Methodology. The adopted development model was a concurrent model: For the Frontend development the spiral model was used, which facilitates its design through the stages of prototyping, analysis and design. On the other hand, for the development of the Backend, the waterfall model was used, given the nature of the project, where the requirements are pre-established and with few changes. The phases of this model are: (Requirements. Survey of requirements, Design. Development of the database and class diagrams, Implementation Coding, Check. Tests, Maintenance. Correct errors in functionality) Contribution. Implementation of project-based learning (PBL) as a motivational teaching strategy in the development of web applications. Use of robust development tools used today by software factories, such as: Java, Spring, HTML5, CSS3, and Apache Maven; all this under a Model View Controller architecture.
\end{abstract}

Project-based learning, ICT, Web development

Citación: TREJO-CUEVAS, Apolinar, PARRA-RODRÍGUEZ, Gerardo, DÍAZ-ROBLEDO, María Teresa y TORRESYÁÑEZ, Javier Jesús. ZonaTIC: Sitio web para la divulgación de la ciencia de las TIC en pro de la inclusión digital. Revista de Tecnologías de la Información. 2019. 6-20: 14-18

\footnotetext{
* Correspondencia del Autor (apolinartrejo@utng.edu.mx)

$\dagger$ Investigador contribuyendo como primer autor.
} 


\section{Introducción}

Son numerosos los cambios que afronta el mundo hoy en día en el sector de las Tecnologías de la Información y Comunicación (TIC), al grado de que si no nos informamos oportunamente podemos quedar en poco tiempo obsoletos, o llegar al extremo del analfabetismo informático. A pesar de que las TIC han hecho posible el acercar lugares distantes, también han acentuado aún más la separación de los países desarrollados y menos privilegiados, el surgimiento de la brecha digital. (Espinoza, 2010)

La brecha digital, como lo define Serrano y Martínez (2002), es:

La separación que existe entre las personas (comunidades, estados, países ...) que utilizan las Tecnologías de la Información y la comunicación (TIC) como una parte rutinaria de su vida diaria y aquellas que no tienen acceso a las mismas y que, aunque las tengan no saben cómo utilizarlas. (p. 16)

El gobierno del estado de Guanajuato, en la pasada administración (2014-2018), con el programa Impulso para la Inclusión Digital, ha establecido las bases para atender esta problemática, dotando de tabletas electrónicas tanto a alumnos de educación media y superior, como a docentes, para la consulta, consumo y acceso a la información.

El objetivo general del Programa de Impulso para la Inclusión Digital consiste en proveer una herramienta que auxilie en la consulta, consumo y acceso a la información para la inclusión digital de los estudiantes del primer año del nivel medio superior y primer año del nivel superior, así como otorgar una herramienta de actualización en las tecnologías de la información a los docentes del nivel básico, medio superior y educación normal.(EDUCAFIN, 2019)

Sin embargo, las TIC siguen evolucionando a grandes pasos, con la industria 4.0 se vienen todo un cúmulo de nuevos conceptos tecnológicos, que hacen necesario adoptar nuevas estrategias para lograr dicha inclusión digital.
En el marco de la segunda edición del IT Masters Forum, la subsecretaría de Comunicaciones y Desarrollo, Salma Jalife Villalón: la inclusión digital en México en la Cuarta revolución industrial es una realidad.

Gonçalves (2019) establece que:

La comunicación y divulgación de la ciencia es un pilar fundamental para entender el proceso de transformación social y económica de un país, ya que la generación de nuevos conocimientos, no pueden traer beneficios a la sociedad, si éstos se mantienen restringidos a un ambiente exclusivamente científico. (p. 223)

Lapa y Pretto (2019), establecen la necesidad de construir redes con el fin de diseminar la información y producción de conocimiento colaborativo, particularmente referente a las TIC. (p. 33)

Guerrero (2019), afirma que:

El conocimiento científico que se va generando en los diferentes ámbitos, pero principalmente en el público, requiere de darse a conocer a la sociedad para contribuir tanto en su formación como en su mejor comprensión de los fenómenos. Se sabe que este tipo de conocimiento solo se adquiere en un $5 \%$ en la educación formal. (p. 51)

Para Rizo (2019), las redes de docentes son un espacio factible para realizar trabajos colaborativos, aprovechando el recurso del internet para el desarrollo de materiales virtuales (talleres, cursos). (p. 315) Como vemos, son varias voces las que nos señalan la necesidad de que las universidades adopten un papel protagónico en la comunicación y divulgación de la ciencia de TIC en beneficio de su comunidad y de la sociedad en general.

El presente trabajo corresponde al proyecto ZonaTIC, el cual tiene como objetivo: Implementar un sitio Web que permita establecer una red de colaboración de docentes para divulgar la ciencia de Tecnologías de la Información y Comunicación al personal de la comunidad universitaria de la UTNG en pro de la inclusión digital, aprovechando la infraestructura con la que cuenta la universidad, así como su capital humano. 
El proyecto consta de dos fases:

Fase 1. Desarrollo de un sitio Web (ZonaTIC) que permita la creación y gestión de artículos de divulgación de la ciencia de TIC; motivo del presente trabajo.

Fase 2. Implementación del sitio web a través de la participación de la comunidad UTNG.

\section{Descripción del método}

El modelo de desarrollo adoptado fue un modelo concurrente: modelo espiral y modelo de cascada.

Para el desarrollo del proyecto se hizo uso del modelo de cascada, dada la naturaleza del mismo, en donde los requerimientos son preestablecidos y con pocos cambios. Las fases de este modelo son:

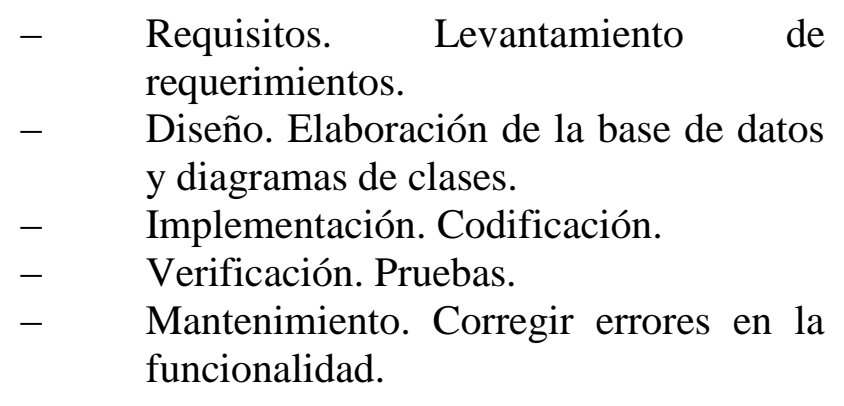

Para el diseño del Frontend se empleó el modelo espiral, el cual facilita su diseño por medio las etapas de elaboración de prototipos, análisis y diseño.

Integración de equipos de trabajo, implementando el aprendizaje basado en proyectos (ABP) como estrategia motivadora.

Los equipos de trabajo se conformaron con alumnos del área de TIC, de los programas académicos de TSU en Tecnologías de la Información y Comunicación área Infraestructura de Redes Digitales, y área Desarrollo de Software Multiplataforma, como parte de los trabajos de la asignatura Integradora I correspondiente al tercer cuatrimestre.

\section{Desarrollo}

Análisis de requerimientos. En este paso se detallan los requerimientos de la aplicación, por medio de entrevistas al cliente.

A continuación, se listan los requerimientos principales:
1) El sitio web deberá permitir administrar y publicar artículos con conocimientos generales y especializados del área de TI.

2) El sitio deberá permitir la autentificación de los usuarios, lo cuales podrán hacer agregar, consultar, modificar y eliminar artículos (de su autoría).

3) Así mismo, otro grupo de usuarios "revisores", podrán hacer revisiones a los artículos y realizar comentarios de retroalimentación a los mismos, hasta su aprobación para su publicación.

4) Los artículos publicados deberán poder consultarse de acuerdo a las siguientes categorías: Conocimientos generales. Engloba los artículos de conocimientos básicos y generales sobre las tecnologías de TI. Conocimientos especializados. Artículos especializados del área, tales como bases de datos, lenguajes de programación, internet de las cosas, etc. TI a la Vanguardia. Artículos de sobre innovaciones tecnológicas, tecnologías emergentes, etc. Eventos TICs. Artículos de divulgación de hechos y actividades referentes al área de TI desarrollas por la comunidad universitaria.

5) El sitio deberá registrar estadísticas de visitas de manera general y por artículo.

\section{Diseño Frontend}

Es la parte de un sitio web que interactúa con los usuarios, por eso decimos que está del lado del cliente. Las herramientas usadas en esta parte de la aplicación son:

\section{- Hypertext Markup Languaje (HTML5). Es el lenguaje usado para crear páginas web. \\ - Cascading Style Sheets (CSS3). En diseño web, la apariencia de la página en el navegador está controlada por las reglas de estilo escritas en CSS. \\ - JavaScript. Es un lenguaje que agrega interactividad y comportamiento a las páginas web. \\ Bootstrap. Es una biblioteca multiplataforma o conjunto de herramientas de código abierto para diseño de sitios y aplicaciones web.}

\section{Desarrollo Backend}

Es la parte del desarrollo web que se encarga que todo funcione; conecta con la base de datos y el servidor que utiliza dicho sitio web.

TREJO-CUEVAS, Apolinar, PARRA-RODRÍGUEZ, Gerardo, DÍAZROBLEDO, María Teresa y TORRES-YÁÑEZ, Javier Jesús. ZonaTIC: Sitio web para la divulgación de la ciencia de las TIC en pro de la inclusión digital. Revista de Tecnologías de la Información. 2019 
Las herramientas usadas en esta parte de la aplicación fueron:

- MySQL. Gestor de bases de datos.

- Apache Tomcat. Servidor de aplicaciones.

Java (JSP). Permite la creación de páginas dinámicas.

Spring. Es un framework para el desarrollo de aplicaciones.

Maven. Es una herramienta de software para la gestión de proyectos Java.

MVC. Es un patrón de arquitectura de software.

\section{Resultados}

Se cuenta con 2 prototipos funcionales los cuales cubren el $80 \%$ y $90 \%$ de la funcionalidad requerida.

Se cuenta con capacitación docente y experiencia adquirida en el uso de herramientas de vanguardia para el desarrollo de aplicaciones web.

Se está haciendo uso de la infraestructura disponible del área (un servidor), para alojar las aplicaciones y permitir el trabajo colaborativo.

Se ha aprovechado la sinergia con empresas vinculadas con el área, para utilizar herramientas de vanguardia.

\section{Conclusiones}

Aunque de momento no se ha logrado obtener un proyecto concluido al $100 \%$, la experiencia adquirida ha sido muy enriquecedora.

Uno de los mayores problemas con los que se enfrentaron los equipos de trabajo fue la puesta a punto de las herramientas de desarrollo, dada las diferentes versiones existentes, así como los requerimientos de las mismas.

La arquitectura adoptada (MVC) permite que la aplicación sea escalable y vaya creciendo en los módulos de la misma.

Por otra parte, se está aprovechando la infraestructura disponible del área (un servidor), para alojar las aplicaciones y permitir el trabajo colaborativo, actividad fundamental en el trabajo profesional.
Una vez concluida la primera fase, el desarrollo de la aplicación web, se procederá a la segunda fase del proyecto, la elaboración de artículos de divulgación de temas de TI, con la participación de la comunidad universitaria, para lo cual será necesario establecer un comité revisor para que garantice la calidad de los trabajos, promover el uso de sitio ZonaTIC, y evaluar su impacto.

Por otra parte, en vista de todo lo mencionado en el presente artículo, la universidad tiene una gran oportunidad de desarrollar un papel protagónico en la divulgación de temas tecnológicos en beneficio de su comunidad y de la inclusión digital.

La Tecnologías de la Información y Comunicación seguirán evolucionando por muchos años más, y con ello la amenaza de la brecha digital amenaza seguirá incrementándose día a día.

Sin embargo, podemos apoyar a cerrar esa brecha digital a través de la formación de redes de colaboración que apoyen en la divulgación de las TIC haciendo más asequible el conocimiento a la comunidad, permitiendo con ello la generación de habilidades digitales necesarias para la inclusión digital.

\section{Referencias}

EDUCAFIN. (2019). Apoyos en especie: Programa a la inclusión digital a través de entrega de tabletas y programa de entrega de uniformes escolares. Recuperado de http://www.educafin.com/beca/19/tabletas

Espinoza, N. (2010). "La brecha digital. Avances para la superación en Venezuela", Revista Interamericana CTS, (13), 1-16.

Gonçalves, T. S. (2019). Comunicación y divulgación de ciencia en Brasil: un estudio de la usabilidad del portal MCTIC. Miguel Hernández Communication Journal, (10), 223237.

Guerrero, M. V. (2019). Las radios universitarias de México y sus estrategias para comunicar la ciencia en Internet. Anuario electrónico de estudios en Comunicación Social" Disertaciones", 12(2), 50-64. 
Lapa, A., y Pretto, N. D. L. (2019). La comunicación en disputa: el rol de educadores y científicos. REDU. Revista de Docencia Universitaria, 17(1), 33-43.

Rizzo, K. A. (2019). MatemaTICambios: la OEI y el desafío de enseñar en la era digital. Revista Iberoamericana de Ciencia, Tecnología y Sociedad-CTS, 14(41).

Serrano, A. y Martínez, E. (2002). La brecha digital. Mitos y realidades. Mexicali: Universidad Autónoma de Baja California. 